\title{
Soliton Interaction with an External Traveling Wave
}

\section{Gil Cohen}

Racah Institute of Physics, Hebrew University of Jerusalem, Jerusalem 91904, Israel

The dynamics of soliton pulses in the Nonlinear Schrödinger Equation (NLSE) driven by an external Traveling wave is studied analytically and numerically. The Hamiltonian structure of the system is used to show that, in the adiabatic approximation for a single soliton, the problem is integrable despite the large number of degrees of freedom. Fixed points of the system are found, and their linear stability is investigated. The fixed points correspond to a Doppler shifted resonance between the external wave and the soliton. The structure and topological changes of the phase space of the soliton parameters as functions of the strength of coupling are investigated. A physical derivation of the driven NLSE is given in the context of optical pulse propagation in asymmetric, twin-core optical fibers. The results can be applied to soliton stabilization and amplification. 


\section{INTRODUCTION}

Investigations of the externally driven Nonlinear Schrödinger (NLS) equation date back to the seminal work of Kaup and Newell [1] on the AC-driven damped NLS equation. That work was also one of the pioneering papers in which a perturbation method for solitons based on the Inverse Scattering Transform (IST) technique was developed. Externally driven NLS equation arises in many applications, mainly in the context of solid state physics, such as long Josephson junctions [2] and charge density waves [3. The same equation describes plasmas driven by RF fields [4]. Much attention has been paid to the study of chaotic phenomena in the phase space of the soliton parameters of the driven system [5]7], and to the formation and stability of soliton states unique to the driven system [7,8]. Problems of generation of solitons via a coupling to external perturbations have been also investigated, for a homogeneous AC-drive [9] and traveling waves 10 .

In the model system that we shall consider (see Eq. (1) below) the perturbation term does not introduce dissipation (the system remains conservative), and the uniform time-dependent driving is generalized to a, spatially dependent, driving field. The physical background of our model system is the nonlinear pulse propagation in optical fibers. In this case the driving force can assume, as will be shown in Sec. IV, the form of a Traveling wave coupled linearly to the NLS equation. The model system can be realized in the context of propagation of envelope optical pulses in twin-core optical fibers. The aim of this work is to study the effects of the driving field on the dynamics of the NLS-solitons. The emphasis is on weak couplings, and the main motivation is stabilizing and controlling solitons by an external traveling wave.

We will limit ourselves to one-soliton pulses (that persist in an unperturbed NLS equation) and investigate the evolution of the parameters of the soliton when it is driven by a Traveling wave. This form of coupling preserves the Hamiltonian structure of the equation, thus making possible to employ Hamiltonian perturbation methods. We will show that, in the adiabatic approximation for a single soliton, the problem is integrable despite the large number of degrees of freedom. We will show that there exists a resonance, or phase locking, between the soliton and the driving field. We will see how this resonance is linked to the particle-like properties of solitons and how the resonance conditions are influenced by the strength of interaction.

Transmission of solitons over long distances is essential for the use of solitons as digital bits in optical transmission lines [11]. This has been achieved by use of Iridium doped fiber amplifiers [12]. Here we will suggest an alternative scheme that employs a twin-core optical fiber. For identical fibers with pulses centered around the same central frequency, the twin-core system is also termed the Nonlinear Directional Coupler [13], and this system has been extensively investigated (see [14] and references therein). Asymmetric twin-core optical fibers were also investigated. Numerical investigation of the asymmetric coupler as a means of performing logical gate operations with solitons was carried out in [15]. In [16], "static" soliton states which can exist in these fibers with a limited asymmetry, were investigated in the model of two coupled NLS equations, where new types of solitons unique to the twin-core fiber were found.

In our model, the two coupled NLS equations, which describe the evolution of optical pulses in asymmetric twin-core fibers, are reduced to a single NLS equation driven by an external traveling wave. We will see how the driving by an external wave can be used to stabilize soliton propagation in optical fibers, suggesting that this scheme can be used in transmission of solitons over long distances. We will also show how solitons can be amplified by the external Traveling wave with slowly varying parameters.

The paper is organized as follows. In Sec. I 1 we outline the Hamiltonian perturbation method for the NLS equation driven by an external traveling wave. In Sec. III we show that the reduced problem is integrable and investigate a novel type of resonance (phase locking) between the soliton and the external wave. The linear stability analysis of this resonance is performed, and the structure and topological changes of the phase plane are investigated, analytically and numerically. A physical derivation of the driven NLS equation and possible applications are presented in Sec. IV. Sec. $\mathrm{D}$ summarizes our results.

\section{HAMILTONIAN PERTURBATION METHOD FOR THE DRIVEN NLS EQUATION}

Consider the NLS equation coupled to an external traveling wave field:

$$
i \frac{\partial \psi}{\partial t}+\frac{\partial^{2} \psi}{\partial x^{2}}+2|\psi|^{2} \psi=\varepsilon \exp [i(k x-\omega t)]
$$

where we use dimensionless variables (see Sec. IV for the corresponding physical units in the case of optical fibers). In Eq. (11) $\varepsilon$ is the (normalized) strength of the coupling, and it is assumed to be small: $\varepsilon \ll 1$. Also, $\omega$ and $k$ are the 
(normalized) frequency and wave number of the driving field, respectively. For specific physical models $\omega$ and $k$ are related by an appropriate dispersion relation.

The unperturbed $(\varepsilon=0)$ version of Eq. (11) is completely integrable [17], and its most interesting solutions are solitons. The full one-soliton solution is given by:

$$
\psi_{\mathrm{sol}}(t, x)=\frac{\frac{\rho}{2} \exp \left[i\left(\frac{p}{2} x+\frac{\left(\rho^{2}-p^{2}\right)}{4} t-\hat{\varphi}-\frac{\pi}{2}\right)\right]}{\cosh \left[\frac{\rho}{2}\left(x-p t-\frac{2 \hat{q}}{\rho}\right)\right]},
$$

where $\rho, p, \hat{q}$ and $\hat{\varphi}$ are free parameters. The quantity $\rho$ defines the amplitude and width $(1 / \rho)$ of the soliton, $p$ is the soliton's velocity, $2 \hat{q} / \rho$ is the location of its "center of mass", and $\hat{\varphi}$ is its initial phase.

Eq. (11) has Hamiltonian form, as it can be obtained from the variational derivative of a Hamiltonian:

$$
\frac{\partial \psi}{\partial t}=-i \frac{\delta H}{\delta \bar{\psi}}
$$

where the Hamiltonian is given by

$$
H[\psi]=\int_{-\infty}^{\infty}\left(\left|\frac{\partial \psi}{\partial x}\right|^{2}-|\psi|^{4}+2 \varepsilon \Re(\bar{\psi} \exp [i(k x-\omega t)])\right) d x .
$$

Here $\bar{\psi}$ is the complex conjugate of $\psi$, and $\Re$ and $\Im$ are the real and imaginary parts of a complex number.

For the unperturbed system, $\varepsilon=0$, the four parameters of the single-soliton solution (2) form a Hamiltonian dynamical system [18]. For the variables $q=\hat{q}-\frac{\rho p}{2} t$, and $\varphi=\hat{\varphi}-\frac{\left(\rho^{2}-p^{2}\right)}{4} t$, the soliton amplitude $\rho$ becomes the canonical momentum conjugate to the coordinate $\varphi$ while the soliton velocity $p$ is the canonical momentum conjugate to $q$. The (reduced) one-soliton Hamiltonian of the unperturbed system is given [18] by:

$$
H=\frac{1}{4}\left(\rho p^{2}-\frac{1}{3} \rho^{3}\right)
$$

Going back to the perturbed system, $\varepsilon \neq 0$, we will employ the adiabatic approximation and investigate slow variations of the soliton parameters caused by the external field. In doing so we neglect radiation effects and possible formation of other solitons. This approach is physically motivated in the context of interaction of soliton pulses in optical fibers, where a typical initial condition is a single soliton solution of the unperturbed NLS equation. It is known that solitons are robust objects, especially in the case when the perturbations to the integrable system are Hamiltonian [19]. Therefore, for weak couplings, it is reasonable to expect that the main effect will be that of the solitons persisting, but slowly changing their parameters.

It is advantageous to preserve the important Hamiltonian properties of the perturbed problem. Therefore, we will treat the perturbed system as a Hamiltonian system in the phase space of the one-soliton parameters driven by the external field. The driving term is obtained directly from the Hamiltonian (何). Indeed, by inserting the unperturbed solution $\psi_{\text {sol }}$ with time-dependent parameters from Eq. (2) into the Hamiltonian (4), we obtain:

$$
H\left[\psi_{\mathrm{sol}}\right]=H(\rho, p, \varphi, q, t)=\frac{1}{4}\left(\rho p^{2}-\frac{1}{3} \rho^{3}\right)+\varepsilon \frac{\pi \sin \left[\frac{(2 k-p) q}{\rho}-\omega t+\varphi\right]}{\cosh \left[\frac{(2 k-p) \pi}{2 \rho}\right]} .
$$

The Hamiltonian (6) describes a dynamical system with two and a half degrees of freedom, as it is nontrivially coupled to an explicitly time-dependent driving force. As the dependence of the Hamiltonian upon $\varphi$ and $t$ enter only through a linear combination of these variables, a simple canonical transformation (see below) will eliminate the explicit time dependence, thus yielding an integral of motion: the new Hamiltonian. The new Hamiltonian with two degrees of freedom may still seem non-integrable, and one is tempted to look for chaos in this system. We will show, however, that because of the existence of an additional integral of motion, the one-soliton problem is actually integrable and can be fully investigated analytically. Formally, integrability occurs for any $\varepsilon$. We expect, however, that the adiabatic approximation (that neglects radiation and possible creation of other solitons) will be valid only for small enough $\varepsilon$. Therefore, we will treat the driving term perturbatively and correspondingly require that $\varepsilon \ll 1$. On the other hand, one should assume that $\rho>1$, to make the contribution of the nonlinear term in the NLS equation significant. This means that $\rho \gg \varepsilon$ which will be assumed to hold in the following. 


\section{PERTURBED ONE-SOLITON PHASE SPACE: INTEGRABILITY AND RESONANCE}

It can be easily checked that the perturbed one-soliton Hamiltonian (6) has an additional integral of motion,

$$
\rho(p-2 k)=\text { const } .
$$

This integral follows from the complete, irreduced system, Eq. (1), possessing an exact conservation law:

$$
I[\phi]=\Im \int_{-\infty}^{\infty}\left(\phi \frac{\partial \bar{\phi}}{\partial x}\right) d x=\text { const }
$$

where $\phi=\psi \exp [-i(k x-\omega t)]$. Indeed, inserting the one-soliton Ansatz (2) for $\psi$ in Eq. (8), one immediately obtains Eq. (7).

Note that the single-soliton solution form, Eq. (7) in our case, of exact integrals of the type (8), is only an approximation, valid as long as generation of radiation and formation of other solitons are ignored. Even so, it is a good approximation [19, 14, when studying single soliton evolution under Hamiltonian perturbations.

We now choose the integral of motion (77) as a new momentum and make the corresponding canonical transformation in the one-soliton parameters' phase space. Simultaneously, we exploit the abovementioned symmetry in the time dependence of the one-soliton Hamiltonian and introduce the new phase $\Phi$ (see below). The generating function of this canonical transformation is:

$$
S(\rho, p, \Phi, Q)=-(p-2 k) \rho Q-\rho\left(\Phi+\omega t-\frac{\pi}{2}\right) .
$$

Therefore, the new canonical coordinates are given by

$$
\begin{array}{rlrl}
R & =\rho, & & P=\rho(p-2 k), \\
\Phi=\varphi-\frac{(p-2 k)}{\rho} q-\omega t+\frac{\pi}{2}, & & Q=q / \rho .
\end{array}
$$

In the new coordinates the Hamiltonian becomes

$$
H(R, P, \Phi, Q)=\frac{1}{4}\left[\frac{1}{R}(P+2 R k)^{2}-\frac{1}{3} R^{3}\right]-\omega R+\varepsilon \frac{\pi \cos \Phi}{\cosh \left(\frac{\pi P}{2 R^{2}}\right)},
$$

and the new momentum $P$ is a constant of motion of the system. Therefore, the Hamiltonian (11) represents a system with one effective degree of freedom and is therefore integrable. This fact excludes any possibility of chaotic motion 20] in the reduced, one-soliton system.

Let us investigate possible resonances between the external wave and the soliton. In the language of the Hamiltonian (11), an exact resonance is related to a stable (elliptic) fixed point. Looking for fixed points, we should equate $\partial H / \partial R$ and $\partial H / \partial \Phi$ both to zero. This yields two conditions. The first is

$$
\Phi_{0}=\pi n \quad n=0,1,
$$

an exact condition, valid in all orders of $\varepsilon$. Writing down the second condition, we will first limit ourselves to the zero order approximation with respect to $\varepsilon$ :

$$
R_{0}= \pm\left[2\left(k^{2}-\omega\right) \pm\left(4\left(k^{2}-\omega\right)^{2}-P^{2}\right)^{1 / 2}\right]^{1 / 2}
$$

The non-trivial, multi-valued resonance condition for the momentum $R$ arises from the non-standard dependence of the Hamiltonian (11) on the momentum $R$.

From Eq. (13) we obtain two conditions for the existence of a resonance between the driving field and soliton. First, $k^{2}>\omega$, which is a condition on the dispersion relation of the external wave alone. Second, $4\left(k^{2}-\omega\right)^{2}>P^{2}$, which is a condition on the parameters of the soliton and the external wave.

The linear stability of the fixed points (12) and (13) is determined by the sign of the product $F G$, where $G=$ $\partial^{2} H_{0} / \partial R^{2}\left(R=R_{0}\right)\left(H_{0}\right.$ is obtained from the Hamiltonian (11) by setting $\varepsilon$ to 0$)$, and $F=\varepsilon \pi \cosh ^{-1}\left(\pi P / 2 R_{0}^{2}\right)$

The phase plane of the system (11) for a relatively weak coupling $(\varepsilon=0.3)$ is shown in Fig. 1. The four-valued, in $R$, fixed points are clearly seen. The coordinates of the fixed points, found numerically, agree very well with the values given by Eqs. (12) and (13). 
Fig. 2 shows the phase plane of the system (11) for a stronger coupling, $\varepsilon=0.6$. One can see that a topology of the phase plane has changed. Four out of the eight fixed points have disappeared. In order to explain this bifurcation, we should modify the resonance condition, Eq. (13) and take into account higher order corrections in $\varepsilon$.

In the first order in $\varepsilon$ the resonance condition for $R$ becomes:

$$
R^{4}-4\left(k^{2}-\omega\right) R^{2}+P^{2} \mp \varepsilon \frac{4 \pi^{2} P \sinh \left(\frac{\pi P}{2 R_{0}{ }^{2}}\right)}{R_{0} \cosh ^{2}\left(\frac{\pi P}{2 R_{0}{ }^{2}}\right)}=0,
$$

where the values for $R_{0}$ should be taken form the zeroth approximation (13). From Eq. (14) we can see that indeed not all the fixed points which were present when $\varepsilon$ was small (see Eqs. (12) and (13)) still persist when the coupling is increased. For a given $P$, when

$$
\varepsilon>\frac{P R_{0} \cosh ^{2}\left(\frac{\pi}{2} \frac{P}{R_{0}^{2}}\right)}{4 \pi^{2} \sinh \left(\frac{\pi}{2} \frac{P}{R_{0}^{2}}\right)},
$$

two of the resonant values of $R$ do not exist anymore, thus leading to a topological change in the phase plane, as observed in Fig. 2.

Now let us discuss the resonance condition in physical terms. Transforming back to the "lab" coordinates, using Eq. (10), we can write the resonance condition $\dot{\Phi}=0$ as

$$
\dot{\varphi}-\frac{d}{d t}\left(\frac{(p-2 k) q}{R}\right)-\omega=0 .
$$

Using the definition of $P$ in Eq. (10), Eq. (15) can be rewritten as

$$
\dot{\varphi}-\frac{d}{d t}\left(\frac{P q}{R^{2}}\right)-\omega=0 .
$$

But $P$ is a constant of motion of our reduced system, so $\dot{P}=0$. Also, $\dot{R}=0$ at resonance. Therefore, Eq. (16) reduces to

$$
\dot{\varphi}-\left(\frac{p-2 k}{R}\right) \dot{q}-\omega=0 .
$$

Now, $\dot{q}=R v_{s} / 2$, where $v_{s}$ in the center-of-mass velocity of the envelope of the soliton. Therefore, defining $\kappa \equiv p / 2$ as the soliton internal wave wave number (see Eq. (2)), and $\omega_{c} \equiv \dot{\varphi}$ (in the leading order in $\varepsilon$ ), the resonance condition becomes

$$
\omega_{c}-\kappa v_{s}=\omega-k v_{s}
$$

From Eq. (18) we can see that the resonance described by the reduced Hamiltonian (11) is a Doppler shifted resonance between two waves: the external (pumping) wave with the wave number $k$ and frequency $\omega$, and the carrier wave of the soliton with the wave number $\kappa$ and frequency $\omega_{c}$. It is interesting that there are two Doppler shifts in the resonance condition. The first Doppler shift enters the right hand side of Eq. (18), and it is by the center-ofmass velocity of the soliton. The second Doppler shift, entering the left hand side, is less intuitive, and is also by the center-of-mass velocity of the soliton, but this time with the soliton carrier wave's wave number. This problem provides us with another non-trivial example of particle-like properties of solitons.

Let us look at the limiting case of $k \rightarrow 0$, in which the system should reduce to well known resonance with a homogeneous AC-drive [1]. For $\omega<0$ and $p=0$ (which are the parameter values studied in [i]), the constant of motion $P=0$ and the resonant values of the soliton's amplitude are reduced, see Eq. (13), to $\rho=R= \pm \sqrt{2 \omega}$, as obtained in [1]. For $\omega>0$, we find from Eq. (13) that since $k^{2}>\omega$ must hold there will be no resonance for $k=0$. So that in this case the resonance is unique to a coupling to an external travelling wave.

Another interesting limiting case is when the values of the wave vectors of the driving field and the internal soliton wave are close to each other. That is $k \simeq \kappa=p / 2$. This translates (see the transformation (10)) to $P \rightarrow 0$. From Eq. (14) we can see that in this limiting case the reduced system will be exactly at the bifurcation point, for all values of $\varepsilon$. Note that for $\kappa=0$, also $v_{s}=0$ so that the resonance (18) is reduced to a simple resonance. 


\section{PHYSICAL MODEL AND APPLICATIONS}

In this Section we will present a physical derivation of Eq. (11). We will start with the equations for the envelopes of pulses in the cores of two adjoining, closely spaced, non-identical, single-mode fibers (twin-core optical fibers) [21,22]:

$$
\begin{gathered}
i \frac{\partial \psi_{1}}{\partial x}+\frac{\partial^{2} \psi_{1}}{\partial t^{2}}+2\left|\psi_{1}\right|^{2} \psi_{1}+\delta \alpha_{12} \psi_{2} \exp [-i(\hat{k} x-\hat{\omega} t)]=0, \\
i\left(\frac{\partial \psi_{2}}{\partial x}-\beta_{1} \frac{\partial \psi_{2}}{\partial t}\right)+\beta_{2} \frac{\partial^{2} \psi_{2}}{\partial t^{2}}+2\left|\psi_{2}\right|^{2} \psi_{2}+\frac{\alpha_{21}}{\delta} \psi_{1} \exp [i(\hat{k} x-\hat{\omega} t)]=0 .
\end{gathered}
$$

The coordinates $x$ and $t$ in Eqs. (19) are written in the "soliton units" [23] corresponding to Eq. (19a). The couplings $\alpha_{12}$ and $\alpha_{21}$ result from the overlapping of the evanescent fields of the transverse fiber modes with the fields in the adjoining fiber cores. Since the fibers are not identical, the coupling is not symmetric, i.e. $\alpha_{12} \neq \alpha_{21}$. It is assumed that the transverse fiber mode is not affected by the proximity of the adjoining fiber, and by the identical transverse mode in it. We also assume that the interaction term arising from the cross phase modulation (term proportional to $\left.\left|\psi_{i}\right|^{2} \psi_{3-i}, i=1,2\right)$ can be neglected. $\delta=\left(\gamma_{1} / \gamma_{2}\right)^{1 / 2}$ is the ratio of the nonlinearity strengths in the two fibers, where [24]

$$
\gamma_{i}=\frac{n_{2} \omega_{i}}{c A_{\mathrm{i}}^{\text {eff }}} \quad \mathrm{i}=1,2,
$$

$A_{\mathrm{i}}^{\text {eff }}$ is the effective core area (which scales like $\rho_{\mathrm{i}}^{2}, \rho_{\mathrm{i}}$ being the fiber core radius), $n_{2}$ is the Kerr coefficient, $c$ is the speed of light, and $\omega_{i}$ is the carrier frequency in each fiber. The amplitudes $\psi_{i}$ of the pulses are scaled, following [23], to $\left(\gamma_{i} / \beta\right)^{1 / 2} T_{0}$, where $\beta$ is the dispersion coefficient of the pulses in Eq. (19a), and $T_{0}$ is the pulse width.

It follows from Eq. (20) that the inequality $\delta \neq 1$ may result from the fibers having different radii, in which case $\delta$ is the ratio between the radii of the fiber cores of the two fibers. Also, if the fibers are centered around different central frequencies $\omega_{i}$, then $\delta=\left(\omega_{1} / \omega_{2}\right)^{1 / 2}$. Let us continue our discussion of the different coefficients in Eq. (19). The coefficient $\beta_{1}$ is a measure of the difference in the group velocity in Eq. (19b) from that in Eq. (19a). The coefficient $\beta_{2}$ is the ratio of the dispersion coefficients of the two fibers. $\beta_{2} \neq 1$ may result from the fibers not having the same transverse wave numbers, or from the pulses in the fibers being centered around different central frequencies. The different carrier frequencies and/or transverse wave numbers also lead to the fibers having different phase velocities. This fact results in the oscillatory term in the interaction, with $\hat{k}$ and $\hat{\omega}$ being the mismatches in the wave-number and frequency, respectively. This oscillatory term arises in the evaluation of the overlap integral of the transverse modes of the two fibers. Notice that, as the coupling in Eqs. (19) is asymmetric, it is not possible in general to cast the system in a Hamiltonian form as it was done in [22]. We will show below, however, that in a certain limit the system (19) can be reduced (see Eq. (22)) to an equation possessing Hamiltonian structure.

We assume that the interaction term (the r.h.s.) in Eq. (19a) is much larger than the interaction term in Eq. (19b): $\delta \alpha_{12} \gg \alpha_{21} / \delta$. This condition implies that $\delta \gg 1$ which occurs when the ratio between the radii of the two fibers is large, and when the carrier frequencies are not the same. Under these conditions the interaction term in Eq. (19b) can be neglected, and Eq. (19b) is decoupled from Eq. (19a) in the sense that it only enters as a driving term in Eq. (19a), while there is no back action. Now, if we further assume that the pulses described by Eq. (19b) are in the positive (normal) dispersion regime then there is no modulational instability [24] in Eq. (19b]), and stable linear dispersive waves can propagate in the fiber. We are interested in the small amplitude limit of Eq. (19b), when the pulses are just linear waves. In this case we can drop the term arising from the Kerr nonlinearity. Then the set of equations 19. can be written as:

$$
\begin{gathered}
i \frac{\partial \psi_{1}}{\partial x}+\frac{\partial^{2} \psi_{1}}{\partial t^{2}}+2\left|\psi_{1}\right|^{2} \psi_{1}+\varepsilon \psi_{2} \exp [-i(\hat{k} x-\hat{\omega} t)]=0, \\
i\left(\frac{\partial \psi_{2}}{\partial x}-\beta_{1} \frac{\partial \psi_{2}}{\partial t}\right)+\beta_{2} \frac{\partial^{2} \psi_{2}}{\partial t^{2}}=0
\end{gathered}
$$

where $\varepsilon=\delta \alpha_{12}$. Therefore the system (19) reduces to a single NLS equation driven by an external Traveling wave.

The equation for $\psi_{1}$, Eq. 21a), can be written (omitting the indices) as: 


$$
i \frac{\partial \psi}{\partial x}+\frac{\partial^{2} \psi}{\partial t^{2}}+2|\psi|^{2} \psi+\varepsilon \exp [i(K x-\Omega t)]=0
$$

where the dispersion relation $\Omega(K)$ is given by the equation for $\psi_{2}$ in Eq. (21b) together with the phase mismatch $\hat{k}$ and $\hat{\omega}$ from Eq. (19).

Eq. (22) is equivalent to Eq. (1) with $x$ and $t$ interchanged, $K=-\omega$, and $\Omega=-k$. Therefore we can apply the results obtained in Sec. III to the analysis of the dynamics of soliton pulses in twin-core fibers, under the conditions that lead to Eq. (22).

One application of our results is soliton phase locking. The resonance conditions [see Eqs. (12) and (13)] correspond to the soliton's parameters being phased locked to the driving field. For the stable fixed points this allows fixing of the soliton parameters (see also Fig. 11). Specifically, the soliton amplitude $\rho$, see Eq. (2), is constant. The values of the soliton parameters for which the resonance condition is satisfied are given, using Eq. (10), by Eqs. (12) and (13), and by the value of the constant of motion, Eq. (7), which is determined by the initial conditions of the soliton pulse and by the parameters of the driving field. Furthermore, the resonance is sustained, as can be seen from Fig. 2, also for large values of coupling ( $\varepsilon$ in Eq. (11)), only that the number of resonances is decreased (see Eq. (14)).

The phase locking can be used in order to stabilize solitons. In any real soliton transmission system there exists dissipation due to fiber losses. The dissipation will result in a decrease of the solitons' amplitude. The dissipation can be incorporated into the NLS equation by the addition [24 of a perturbation term in the form $-i \Gamma \psi$. Although the dissipative term cannot be directly included in our Hamiltonian perturbation approach, we can anticipate, in analogy to the problem of a driven damped oscillator, that by keeping the soliton's parameters in resonance with the driving field we will be able to overcome the effect of the losses in the system. In this case, the elliptic fixed points of Figs. 1 and 2 will become attracting points.

Another application for which the coupled system can be used is soliton amplification. The amplitude ( $\rho$ in Eq. (2)) of the soliton can be increased for pulses which parameters correspond to periodic orbits surrounding stable fixed points (see Figs. 1 and 2). The period of oscillations for the stable orbits is long and, for small oscillations, is given by $\varepsilon^{1 / 2} F G$, where $F$ and $G$ were defined following Eq. (13). By a proper choice of the initial conditions and interaction length the soliton amplitude can be increased by performing a half period of nonlinear oscillation around a stable fixed point.

Another mechanism, by which the amplitude of solitons can be increased more significantly, is the "Dynamic Autoresonance" [9, 10,25]. The frequency of nonlinear oscillations depends on their amplitude. Therefore, the infinite growth of the amplitude, obtained for a linear, dissipation free oscillator driven by a resonant external force, is not possible for a nonlinear oscillator with constant parameters. By "chirping" adiabatically the driving field's frequency, one can preserve the phase plane structure so that the oscillator will continue to perform nonlinear oscillations around the (time dependent) resonant value of the action variable, $R$ in Eq. (11) in our case. The soliton parameters will remain phase locked in resonance with the (slowly varying) driving field. Since $R$ is the soliton amplitude, see Eq. (10), this mechanism provides a means for substantially increasing the soliton amplitude in a resonant manner.

\section{SUMMARY}

We have investigated the evolution of single-soliton pulses of the Nonlinear Schrödinger (NLS) equation driven by an external traveling wave field. This system, even though not integrable, is still Hamiltonian. Using the Hamiltonian structure and adiabatic approximation for a single soliton, we reduced the perturbed NLS equation to a two-and-ahalf dimensional Hamiltonian system in the phase space of the parameters of the single soliton solution. One integral of motion in this system results from the fact that the time-dependence drops out in a rotating reference frame. An additional integral of motion is a consequence of an exact integral of motion in the complete, unreduced partial differential equation. Therefore, the reduced system becomes effectively one-dimensional and therefore integrable. Physically, the reduced system represents a nonlinear oscillator (with an unusual form of the Hamiltonian) coupled to an external harmonic force. The phase plane of this system was investigated analytically and numerically, and a good agreement between these two was found. As the coupling strength increases, there occurs a bifurcation in the phase plane of the reduced system. This bifurcation has been explained analytically.

We gave a physical motivation to this model by showing that dynamics of soliton pulses in twin-core, non-identical, single-mode optical fibers can be reduced to a system in which the dynamics in one core are governed by a NLS equation driven by a linear Traveling wave propagating in the adjoining core. In this regime, one can neglect the back action of the nonlinear wave on the linear wave.

Finally, we discussed possible applications of our results to stabilization and amplification of soliton pulses in the asymmetric, twin-core optical fiber. 


\section{ACKNOWLEDGMENTS}

We are extremely grateful to B. Meerson for a continuous interest and advice, and for a critical reading of the manuscript.

FIG. 1. The phase plane of the Hamiltonian system (11) for a weak coupling. The parameters are $\varepsilon=0.3, P=3.0, k=2.0$ and $\omega=2.0$. The resonances corresponding to the four-valued solution of Eq. (13) are clearly seen. Note that the stability of the resonant points is interchanged for positive and negative values of $R$.

FIG. 2. The phase plane of the Hamiltonian system, Eq. (11), for a stronger coupling. $\varepsilon=0.6$, The parameters are the same as in Fig. 1, except that $\varepsilon=0.6$. A bifurcation has occurred. In accordance with Eq. 144, for each of the resonant values of $R$ there are no longer two resonant phases, one for a stable (elliptic) point and one for an unstable (hyperbolic) point. Only one fixed point is left for each value of $R$. The phase plane topology has changed accordingly.

[1] D. J. Kaup and A. C. Newell, Proc. R. Soc. Lond. A 361, 413 (1978).

[2] P. S. Lomdahl and M. R. Samuelsen, Phys. Rev. A 34, 664 (1986).

[3] D. J. Kaup and A. C. Newell, Phys. Rev. B 18, 5162 (1978).

[4] K. Nozaki and N. Bekki, Physica D 21, 381 (1986).

[5] K. Nozaki and N. Bekki, Phys. Rev. Lett. 50, 1226 (1983).

[6] K. Nozaki and N. Bekki, Phys. Lett. A 102, 383 (1984).

[7] I. V. Barashenkov and Yu. S. Smirnov, Phys. Rev. E 54, 5707 (1996) and references therein.

[8] D. Cai, A. R. Bishop, N. Grønbech-Jensen, and B. A. Malomed, Phys. Rev. E 49, 1677 (1994).

[9] L. Friedland and A. G. Shagalov, Phys. Rev. Lett. 81, 4357 (1998).

[10] I. Aranson, B. Meerson, and T. Tajima, Phys. Rev. A 45, 7500 (1992).

[11] L. F. Mollenauer, E. Lichtman, M. J. Neubelt, G. T. Harvey, in Proc. Conf. on Optical Fiber Communications (OFC '93), Opt. Soc. Am., Washington, D. C. (1993); M. Nakazawa, K. Suzuki, E. Yamada, H. Kubota, Y. Kimura, M. Takaya, ibid.

[12] See the review: H. A. Haus and W. S. Wong, Rev. of Mod. Phys. 68, 423 (1996).

[13] S. M. Jensen, IEEE J. Quantum Electron. 18, 1580 (1982).

[14] G. Cohen, Phys. Rev. E 52, 5565 (1995).

[15] C. C. Yang, Optics Lett. 16, 1641 (1991).

[16] B. A. Malomed, I. M. Skinner, P. L. Chu, and G. D. Peng, Phys. Rev. E 53, 4084 (1996).

[17] S. P. Novikov, S. V. Manakov, L. P. Pitaevsky, and V. E. Zakharov, Theory of Solitons. The Inverse Scattering Method (Consultants Bureau, New-York, 1984).

[18] L. D. Faddeev and L. A. Takhtajan, Hamiltonian Methods in the Theory of Solitons (Springer-Verlag, Berlin, 1987).

[19] C. R. Menyuk, Phys. Rev. A 33, 4367 (1986).

[20] B. V. Chirikov, Phys. Rep. 52, 263 (1979).

[21] A. W. Snyder and J. D. Love, Optical Waveguide Theory (Chapman and Hall, London, 1983).

[22] B. A. Malomed, Phys. Rev. E 51,R864 (1995).

[23] L. F. Mollenauer, R. H. Stolen, and J. P. Gordon, Phys. Rev. Lett. 45, 1095 (1980).

[24] G. P. Agrawal, Nonlinear Fiber Optics (Academic, Boston, 1989).

[25] B. Meerson and L. Friedland, Phys. Rev. A 41, 5233 (1990). 
This figure "figure1.jpg" is available in "jpg" format from: http://arxiv.org/ps/patt-sol/9903006v1 
This figure "figure2.jpg" is available in "jpg" format from: http://arxiv.org/ps/patt-sol/9903006v1 